\title{
Numerical simulation of Richtmyer-Meshkov instability
}

\author{
FU Dexun ${ }^{1}$, MA Yanwen ${ }^{1}$, ZHANG Linbo $^{2} \&$ TIAN Baolin ${ }^{1}$ \\ 1. State Key Laboratory of Nonlinear Mechanics, Institute of Mechanics, Chinese Academy of Sciences, \\ Beijing 100080, China; \\ 2. State Key Laboratory of Scientific and Engineering Computing, Institute of Computational Mathematics, \\ Chinese Academy of Sciences, Beijing 100080, China \\ Correspondence should be addressed to Fu Dexun (email: fud@imech.ac.cn) \\ Received January 2, 2004
}

\begin{abstract}
The compressible Navier-Stokes equations discretized with a fourth order accurate compact finite difference scheme with group velocity control are used to simulate the RichtmyerMeshkov (R-M) instability problem produced by cylindrical shock-cylindrical material interface with shock Mach number $M s=1.2$ and density ratio 1:20 (interior density/outer density). Effect of shock refraction, reflection, interaction of the reflected shock with the material interface, and effect of initial perturbation modes on R-M instability are investigated numerically. It is noted that the shock refraction is a main physical mechanism of the initial phase changing of the material surface. The multiple interactions of the reflected shock from the origin with the interface and the R-M instability near the material interface are the reason for formation of the spike-bubble structures. Different viscosities lead to different spike-bubble structure characteristics. The vortex pairing phenomenon is found in the initial double mode simulation. The mode interaction is the main factor of small structures production near the interface.
\end{abstract}

Keywords: R-M instability, direct numerical simulation, shock-interface interaction.

DOI: $10.1360 / 04 z a 0021$

\section{Introduction}

Consider a material interface between two different media. As an incident shock interacts with the material interface the interface becomes unstable due to shock acceleration. The small disturbances at the interface start to grow. This kind of instability is called Richtmyer-Meshkov instability (or simple R-M instability). R-M instability problem can be met in many important practical applications. For example, the propagation of sound boom in turbulent atmosphere, the Inertial Confinement Fusion (ICF), and the explosion of the supernova.

The R-M instability is also a model problem for studying the physical mechanism of fluid motion from instability to turbulence.

The R-M instability is closely related to the R-T (Rayleigh-Taylor) instabil- 
ity. The R-T instability is an interfacial instability between two different media driven by an external force. Both R-M and R-T instabilities have common features, for example, formation and growth of the bubble and spike structures. But the dynamic characteristics of R-M and R-T instabilities are qualitatively different. The R-T instability occurs only in the case when the external force acts from the heavy fluid to the light fluid, whereas for the R-M instability the interface is always unstable whether the shock collides with the interface from the heavy fluid phase to the light fluid phase or vice versa. The physical phenomenon is more complicated for the R-M instability. Much work has been done for studying the R-M instability ${ }^{[1-4]}$, but mainly for the case of interaction between plane shock and plane interface with perturbation and interaction between plane shock and cylindrical interface with single mode perturbation.

In this paper we present the numerical study of cylindrical shock-cylindrical material interface interaction by using the two-dimensional compressible NavierStokes equations (N-S equations) which are discretized with a high order accurate finite difference approximation ${ }^{[5]}$. The shock is going from the heavy outside gas through the interface to the light gas. Some results are presented for the case in which the shock Mach number $M s$ is 1.2 , and the density ratio of the inside light gas to the heavy outside gas is 1:20. The shock refraction, reflection, interaction of the reflected shock with the material interface, and effect of the initial perturbation modes on the R-M instability are investigated.

The numerical method is briefly described first, then the method is validated with some model tests, and finally the method is used to study the R-M instability.

\section{Simulation method}

\subsection{Method description}

A fourth order accurate symmetrical compact difference approximation is used to discretize the viscous terms of the N-S equations, a fourth order accurate compact difference approximation with group velocity control ${ }^{[5]}$ is used to discretize the convection terms, and a three stage R-K method is used in advance of time.

The method consists of computing a difference approximation $F_{j} / \Delta x$ of $\frac{\partial f}{\partial x}$ using the following equation:

$$
\frac{1}{6} F_{j+1}+\frac{2}{3} F_{j}+\frac{1}{6} F_{j-1}+H_{j+\frac{1}{2}}-H_{j-\frac{1}{2}}=h_{j+\frac{1}{2}}-h_{j-\frac{1}{2}},
$$

where

$$
\begin{aligned}
H_{j+\frac{1}{2}} & =\left(\varepsilon_{2}-\varepsilon_{4}\right)_{j+\frac{1}{2}}\left(F_{j+1}+F_{j}\right), \\
h_{j+\frac{1}{2}} & =\frac{1}{2}\left(f_{j}+f_{j+1}\right)-2 \varepsilon_{4, j+\frac{1}{2}}\left(f_{j+1}-f_{j}\right), \\
\varepsilon_{2, j+\frac{1}{2}} & =\left[g_{j+\frac{1}{2}}(\rho)\right]^{3}, \\
\varepsilon_{4, j+\frac{1}{2}} & =\left[1+\gamma_{0} S_{j+\frac{1}{2}}(\rho)\right] g_{j+\frac{1}{2}}(\rho),
\end{aligned}
$$




$$
\begin{aligned}
S_{j+\frac{1}{2}}(\rho) & =\operatorname{sign}\left[\frac{\partial \rho}{\partial x}, \frac{\partial^{2} \rho}{\partial x^{2}}\right], \\
g_{j+\frac{1}{2}}(\rho) & =\frac{1}{2}\left[g_{j}(\rho)+g_{j+1}(\rho)\right], \\
g_{j}(\rho) & =\left[\left|\rho_{j+1}-2 \rho_{j}+\rho_{j-1}\right| /\left(\rho_{j+1}+2 \rho_{j}+\rho_{j-1}\right)\right]^{\frac{1}{2}} .
\end{aligned}
$$

The function $g(\rho)$ is used to control the accuracy and numerical dissipation, while the function $S(\rho)$ is used to control the numerical group velocity and accuracy of the scheme. The difference approximation has fourth order accuracy in the smooth region.

\subsection{Method validation}

In order to see the capability of the method for capturing shocks with high pressure ratio and material interface with high density ratio the following test models are solved.

(i) Steady one-dimensional shock tube problem

The method presented in section 2.1 is used to approximate the steady onedimensional shock tube problem with incoming Mach number $M a=30$. The computed pressure and density distributions are given in fig. 1.
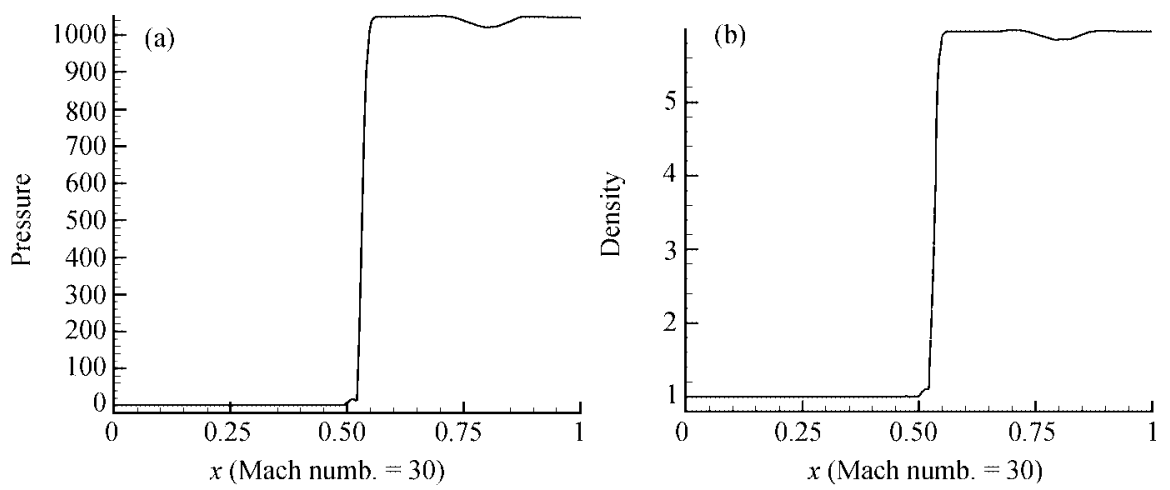

Fig. 1. 1-D shock tube problem. (a) Pressure; (b) density.

(ii) One-dimensional Riemann problem

In fig. 2 are given the results for the one-dimensional Riemann problem in which the initial conditions are as follows:

$$
u 1=u 2=0, \quad p 1 / p 2=10, \quad \rho_{1} / \rho_{2}=800 .
$$

(iii) Two-dimensional Riemann problem

Consider a two-dimensional Riemann problem. The initial distribution is given as follows:

$$
\begin{array}{cccc}
\rho_{1}=1.5, & p_{1}=1.5, & u_{1}=0.0, & v_{1}=0.0 \\
\rho_{2}=0.4670, & p_{2}=0.2228, & u_{2}=1.369, & v_{2}=0.0 . \\
\rho_{3}=0.094, & p_{3}=0.0082, & u_{3}=1.369, & v_{3}=1.369 . \\
\rho_{4}=0.4670, & p_{4}=0.2228, & u_{4}=0.0, & v_{4}=1.369 .
\end{array}
$$



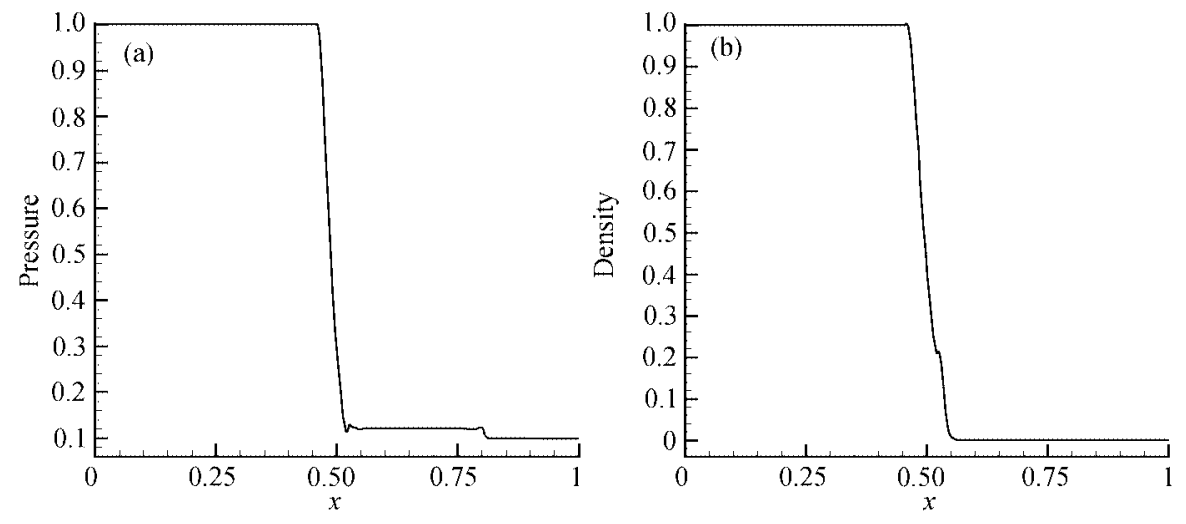

Fig. 2. Solution of 1-D Riemann problem. (a) Pressure; (b) density.

The subscripts in the above equations are the index of the region in which the physical parameters are given (see fig. $3)$.

The computational domain is $0 \leqslant x \leqslant 1,0 \leqslant y \leqslant 1$, and the mesh grid system is $400 \times 400$. The ratio of pressure between regions 1 and 3 is $p_{1} / p_{3}=183$. $\rho, p, u, v$ denote the density, pressure, and velocity components in $x$ and $y$ directions respectively. In fig. 4

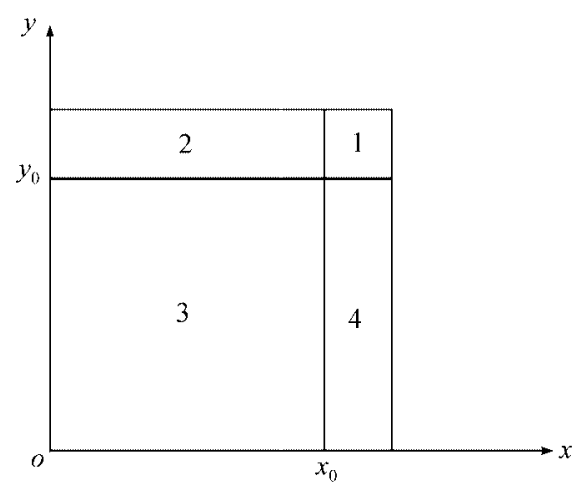

Fig. 3. The 2-D Riemann problem.
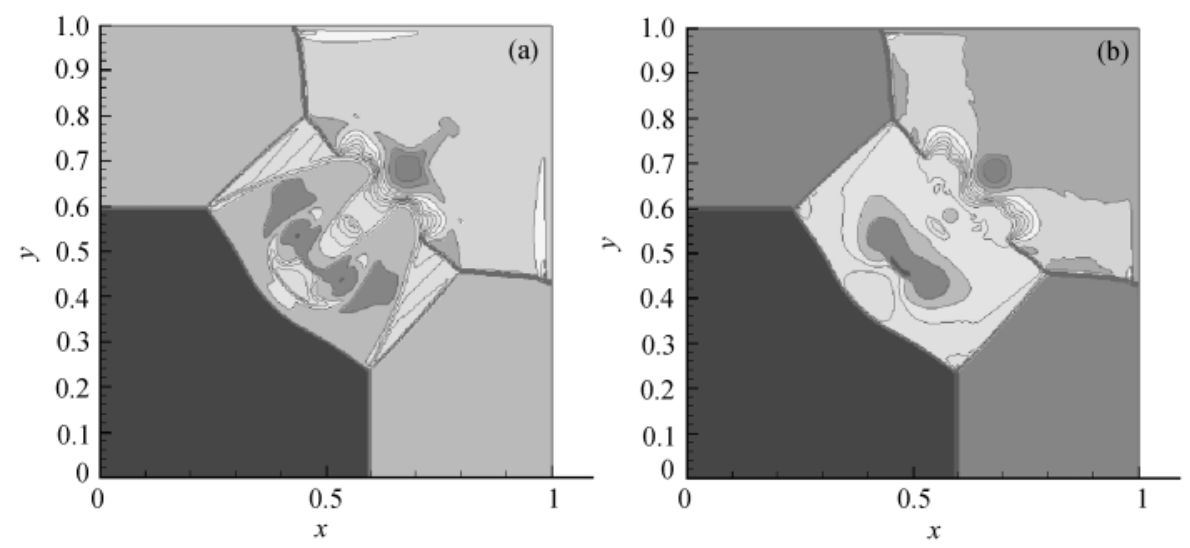

Fig. 4. Solution of a two-dimensional Riemann problem. (a) Pressure contours; (b) density contours. are given the density and pressure contours for two-dimensional Riemann problem with the limit initial conditions satisfying the R-H relation. From these figures we can see the double Mach reflection and contact discontinuity clearly. 
From the above numerical tests we see that the numerical method used in the present paper can capture well the unsteady shock with high pressure ratio and contact discontinuity with high density ratio.

\section{Numerical simulation of R-M instability}

Consider an R-M instability produced by interaction of a cylindrical shock with cylindrical material interface. The shock is going inward from the heavy gas with density $\rho_{1}$ to the light gas with density $\rho_{2}$. This problem is solved by using the two-dimensional compressible N-S equations discretized with the method described in the previous section. A coordinate transformation is used in order to make the mesh finer near the material interface. The interface is tracked with time according to the maximal density gradient $(\operatorname{grad} \rho)_{\max }$. The computational grid is $429 \times 429$.

\subsection{Interaction without surface perturbation}

In order to understand the effect of collision of the refracted and reflected shock with the material interface on compressibility of the light gas we first consider the case of interaction of the cylindrical incident shock with the interface without perturbation. In figs. 5 and 6 are given the results for the case in which the shock Mach number $M s=1.2$, the density ratio $\rho_{1} / \rho_{2}=$ 20 , and the Reynolds number $R e=5000$ and $5 \times 10^{5}$ respectively. The variation of pressure at the cylinder center with time is given in fig. 5. When the shock comes into collision with the interface the discontinuity bifurcates into refracted shock (transmitted shock) going toward the cylinder center, and a reflected wave going outward. The outward propagating wave is a rarefaction wave because the shock collides with the interface from heavy gas to light gas. When the transmitted shock approaches the center the center pressure increases, and then reflected shock is formed. This reflected shock is going outward, and the gas in the central region starts to rarefy with decreasing
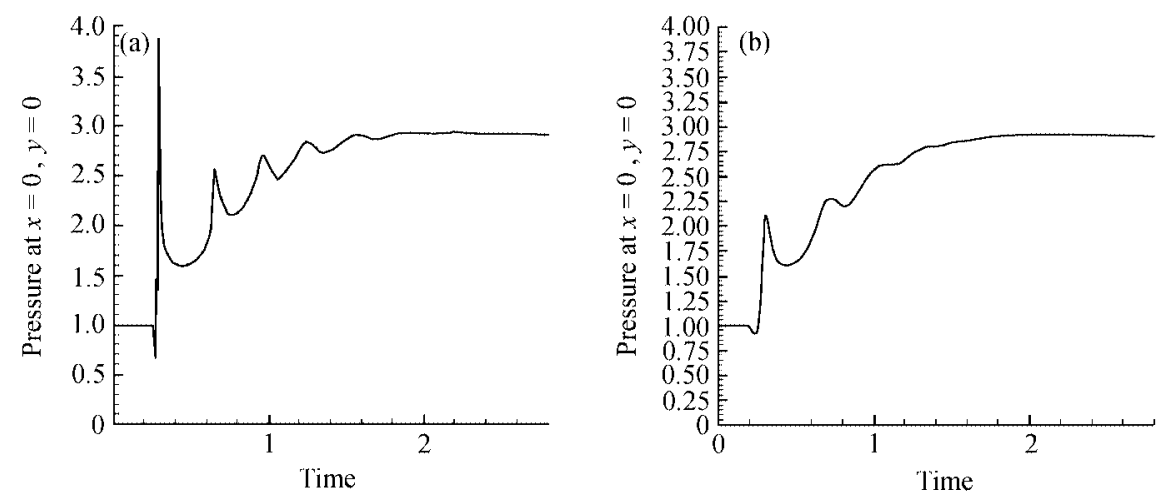

Fig. 5. Variation of central pressure with time (without perturbation). (a) $R e=5 \times 10^{5}$; (b) $R e=5000$. 
pressure. This outward going shock in light media will collide with the interface behind which the gas is heavy. After interaction an inward going reflected shock is formed. The inward going shock will reach the center, then it reflects, and it collides with the interface again. From fig. 5(a) we can see that there are six times of this kind of collision for the case $R e=5 \times 10^{5}$. The strength of the shock becomes weaker and weaker after multiple interaction. In table 1 are given time $t_{1}$ when the shock reaches the center, and time $t_{2}$ when the reflected

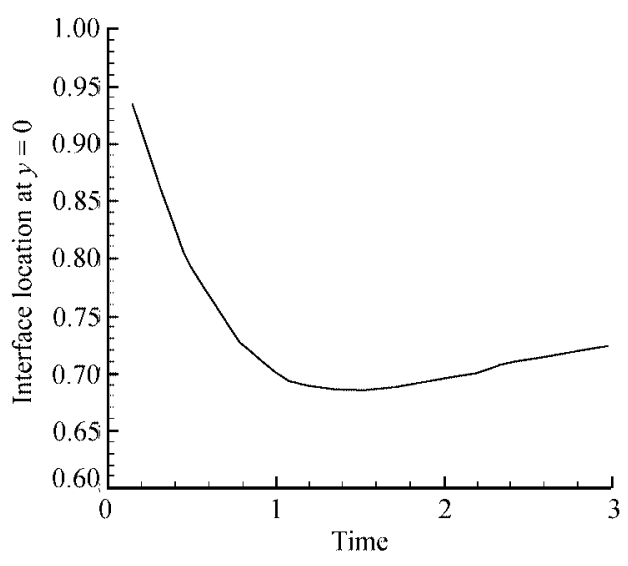

Fig. 6. Variation of interface with time $y=0$. shock collides with the material interface. Comparing fig. 5(a) with fig. $5(\mathrm{~b})$ we see that the viscosity accelerates the shock propagation in the light media and weakens the compressibility. Define the relation $C=r_{\min } / r_{0}$ as a compression ratio to characterize the compressibility of the interior media, where $r_{0}$ is the initial interface radius, $r_{\min }$ is the minimal interface radius after multiple interaction. For the case $R e=5 \times 10^{5}$, we have $C=0.68$ which agrees well with the theoretical result ${ }^{[3]}$. In fig. 6 is shown the variation of interface location with time. We can see that the material interface dilates with multiple collision of the shock with interface.

\begin{tabular}{|c|c|c|c|c|c|c|}
\hline$t_{1}$ & 0.29 & 0.66 & 0.97 & 1.25 & 1.56 & 1.90 \\
\hline$t_{2}$ & 0.43 & 0.75 & 1.06 & 1.34 & 1.70 & 2.02 \\
\hline
\end{tabular}

\subsection{General characteristics of R-M instability}

When the interface is perturbed with small amplitude the interface becomes unstable after collision of the incident shock with the interface. Now we are going to discuss the general characteristics of interface instability based on initial single mode perturbation with small amplitude.

Consider a case with $M s=1.2, R e=5 \times 10^{5}, \rho_{1} / \rho_{2}=20$, and the initial cylindrical surface with perturbation is defined as

$$
r_{0}=1+a \cos (n \varphi), \quad a=0.033, \quad n=12,
$$

where the radius is normalized with the radius of the initial interface without perturbation. The computed results are given in figs. 7-10. Fig. 7 shows the density contours at different time. We see that at $t=1.3$ the discontinuity bifurcates into reflected rarefaction wave and refracted shock after collision with the interface. The pertubation starts to grow linearly, and the interface starts to deform. Existence of pertubation on the interface leads to non-parallelism of the shock gradient $[\operatorname{grad} p]$ with the density gradient $[\operatorname{grad} \rho]$. The vortex production due to this non-parallelism leads to R-M instability. On the other hand, the interface pertubation makes direction changing of the refracted shock 
as shown in fig. 8. As we know the wave refraction rate is $n=c_{2} / c_{1}$, where $c_{1}$, $c_{2}$ are the sound speed inside and outside the material interface. Because the light gas is inside the surface we have $c_{1}>c_{2}$, and $n<1$. According to the Snell law we have $\sin \theta_{i}=n \sin \theta_{r}$, where $\theta_{i}$ is the incident angle and $\theta_{r}$ is the refracted angle. From Snell law we know that in our case the incident angle is less than the refracted angle. From fig. 8 it can be seen that inside the interface near the inward concave region there is compression, and inside the interface near the outward convex region there is rarefaction. This phenomenon can be explained with the optical refraction law. This physical mechanism leads to the change in the phase of the perturbed wave (see fig. $7(\mathrm{~b})$ ), and the stage of nonlinear growth of perturbation starts from the phase changing. The transmitted shock reaches the center and reflects. Collision of this reflected shock with the interface increases nonlinear development of the perturbation (fig. 7(c)). After multiple collisions of the reflected shock with the interface the spike and bubble structures are formed. The spike is a portion of heavy gas penetrating into the light gas, and the bubble a portion of light gas penetrating into the heavy gas. fig. 9 shows variations of density and pressure along the ray of angle $\theta=45^{\circ}$ at different time. From fig. 9 we can see propagation direction of the incident shock, reflected shock and rarefaction wave. From fig. 9(b) we also can see pressure pulsation near the interface for $t \geqslant 0.9$ due to formation of secondary shock ${ }^{[3]}$.
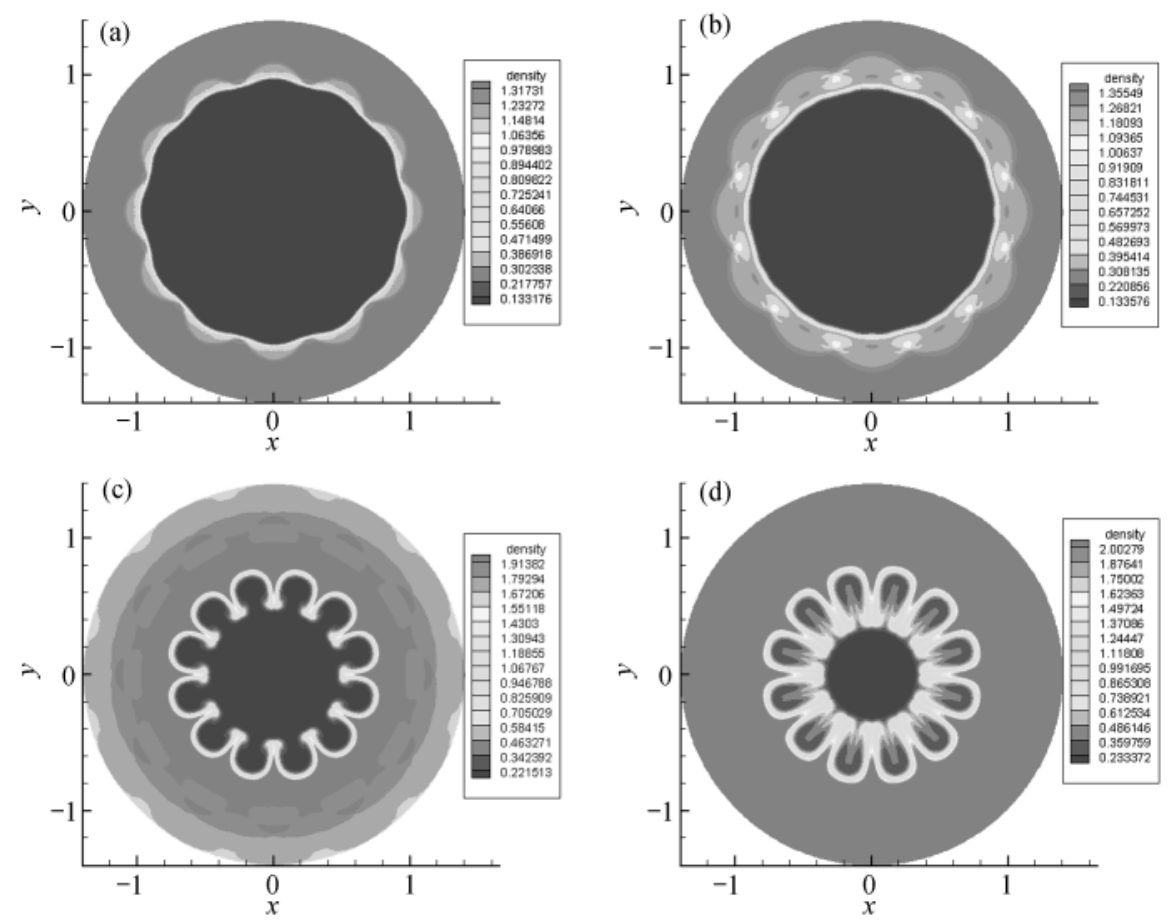

Fig. 7. Density contours at different time (with single mode). (a) Density contours at $t=0.13(M s=1.2, R e=500000) ;(b)$ density contours at $t=0.65(M s=1.2, R e=$ 500000); (c) density contours at $t=2.04(M s=1.2, R e=500000)$; (d) density contours at $t=3.87(M s=1.2, R e=500000)$. 


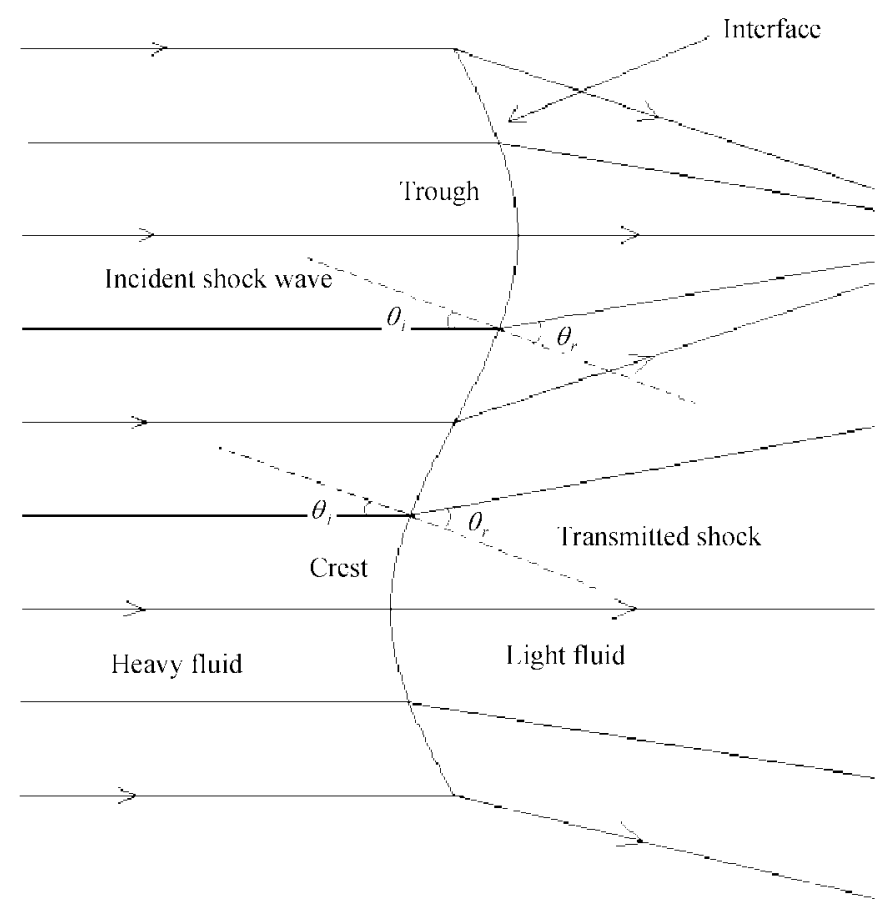

Fig. 8. Schematic diagram of incident and reflected wave.
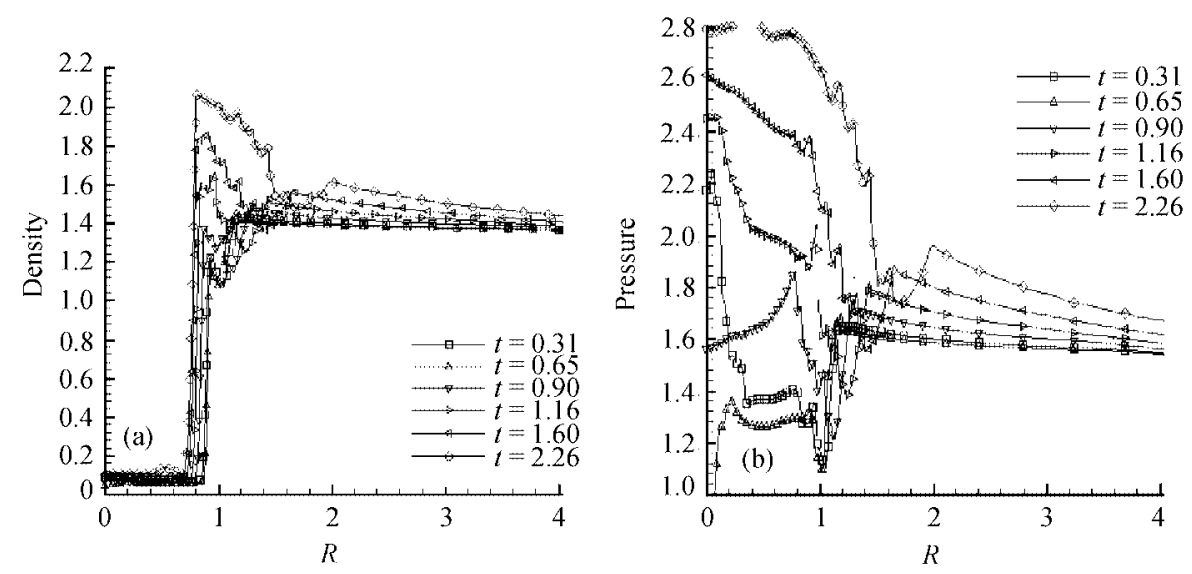

Fig. 9. Density and pressure distribution along the ray $\theta=45^{\circ}$. (a) Density distribution $(M s=1.2, R e=500000)$; (b) pressure distribution $(M s=1.2, R e=500000)$.

\subsection{Effect of initial perturbation on R-M instability}

The cases $M s=1.2, R e=5000, \rho_{1} / \rho_{2}=20$ with both single and double initial interface perturbation modes at interface are computed in order to study the effect of initial perturbation on R-M instability.

The perturbed interface for single mode is the same as in section 3.2. The 
double mode perturbed interface is given as

$$
\begin{aligned}
& r_{0}=1+a\left[\cos \left(n_{1} \varphi\right)+\cos \left(n_{2} \varphi\right)\right], \\
& n_{1}=12, \quad n_{2}=60, \quad a=0.033 .
\end{aligned}
$$

The computed results are given in figs. 10-12. Fig. 10 shows the variation of pressure with time at the cylinder center. By comparing fig. 10(a) with fig. 5(b) we see that the interface instability weakens the effect of compressibility inside the interface from collision of the shock with the interface.
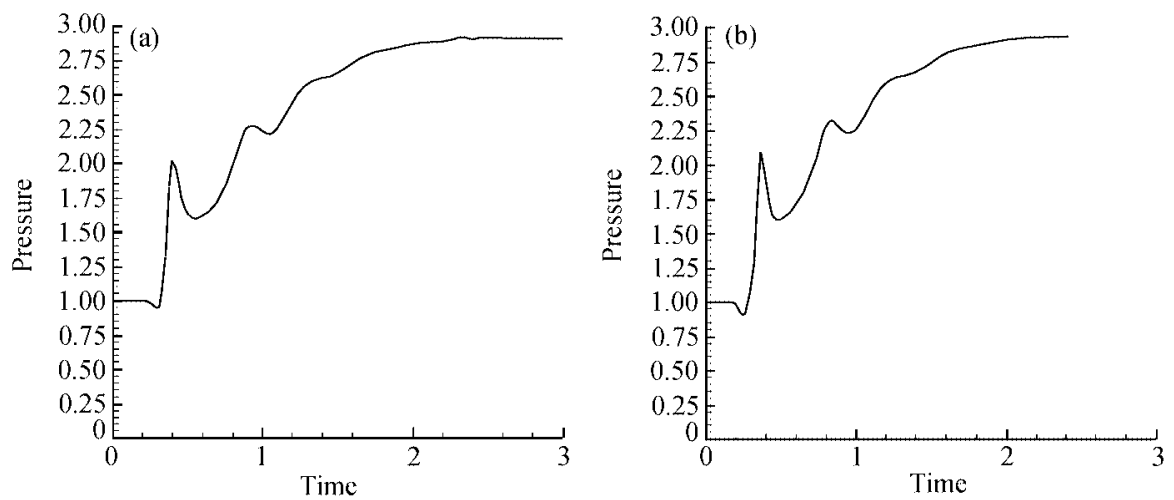

Fig. 10. Variation with time of the pressure at center. (a) Single mode perturbation; (b) double mode perturbation.

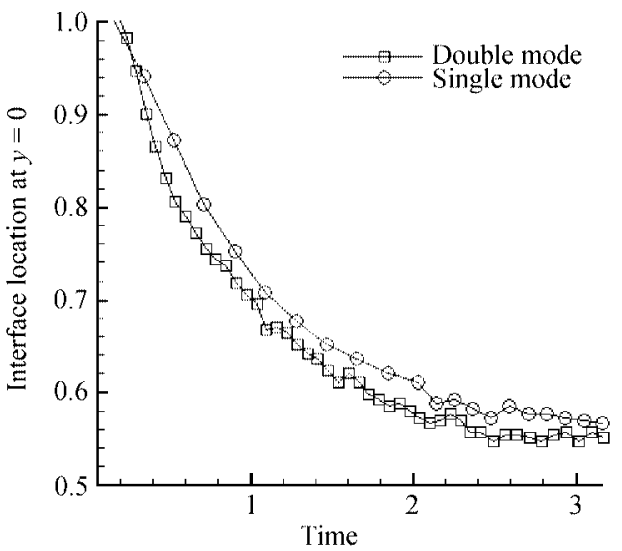

For the case with single mode perturba-

tion four times collisions of reflected shock with the interface can be seen clearly. In fig. 11 is given the variation with time of the interface location. The interface location is defined as $r=\frac{1}{2}\left(r_{\max }+r_{\min }\right)$, where $r_{\min }$ and $r_{\max }$ are the minimal and maximal radial interface location respectively. It can be seen from figs. 10 and 11 that influence of mode competition on propagation speed of the incident and reflected shock in the light medium is small. The times

Fig. 11. Variation of surface location with time. with which the shock reaches the center are listed in table 2 , where $t_{1}$ is for the single mode case and $t_{2}$ is for the double mode case. For the case of single mode perturbation the compression ratio is $C=0.57$, and for the case of double mode perturbation $C=0.54$. As it is noted before, interface instability reduces the effect of compressibility in the light gas region, but mode com-

Table 2 Time when the shock reaches the center

\begin{tabular}{lllll}
\hline$t_{1}$ & 0.40 & 0.95 & 1.32 & 1.78 \\
\hline$t_{2}$ & 0.37 & 0.90 & 1.30 & 1.70 \\
\hline
\end{tabular}



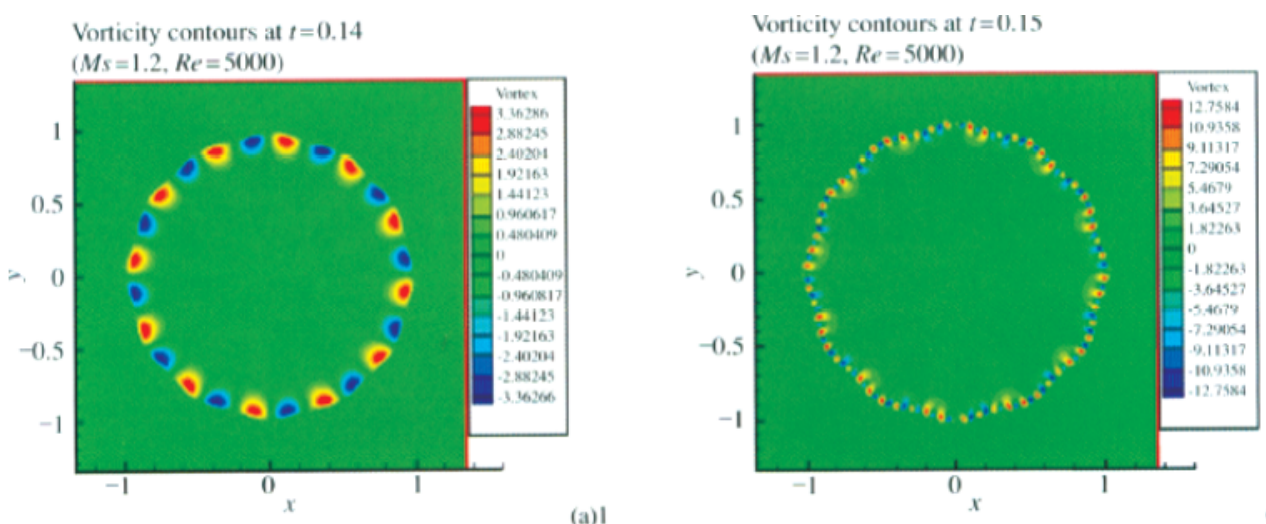

(a)
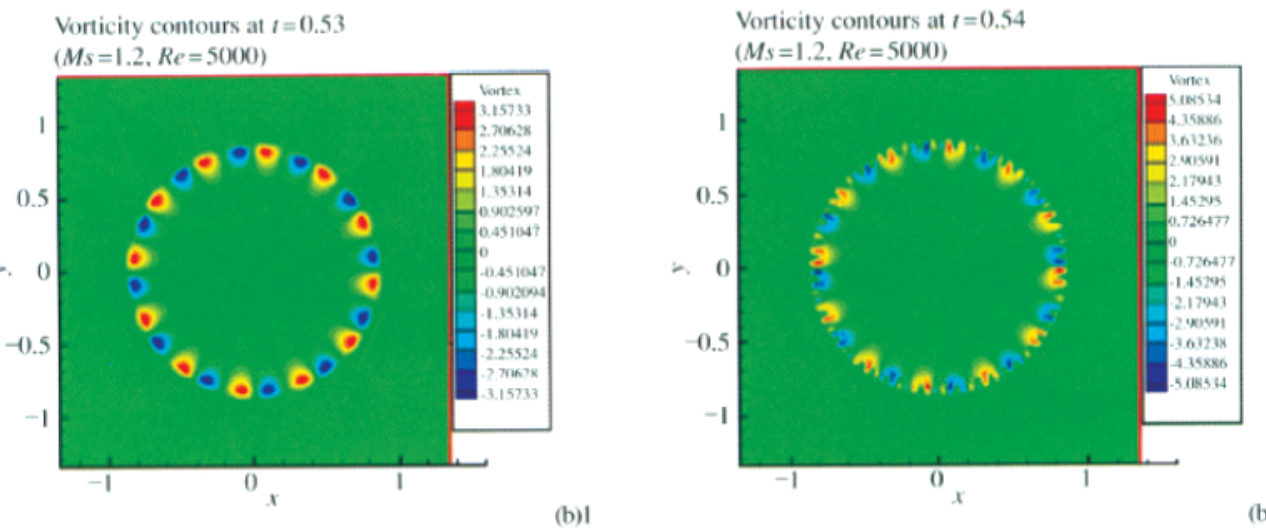

(b)1

Vorticity contours at $t=2.26$ $(M s=1.2, R e=5000)$

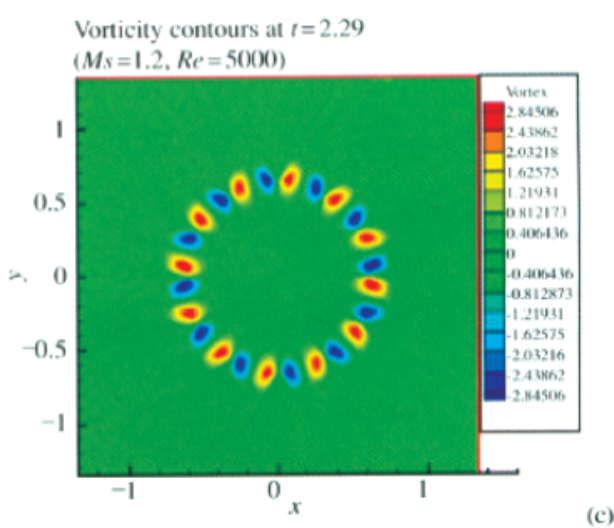

Fig. 12. Vorticity contours for single and double mode initial perturbation at different time. 
petition has small effect on it. Comparing development of interface perturbation we see that the mode competition mainly has effects on the early stage of R-M instability, and its influence on large scale structures is small.

The vorticity contours with two different initial mode perturbation are given in fig. 12. Comparing the results with different initial perturbation modes in fig. 12 (a) 1 and fig. 12(a)2 we see that the initial subharmonic is appended on the basic mode perturbation. With nonlinear growth of perturbation secondary instability occurs, and vortex pairing can be seen in fig. 12(b)1 and fig. 12(b)2. With further development of perturbation we can see array of vortex structures with different strength (fig. 12(c)1 and fig. 12(c)2). The numerical results tell us that the mode competition changes the small structures near the surface, and amplifies the vortex interaction. This is an important factor for stimulating turbulence development.

Acknowledgements This work was supported by the Special Funds for Major State Basic Research Projects (Grant No. G1999032805), National Natural Science Foundation of China (Grant Nos. 10176033, 90205025), and National High-Tech ICF Committee in China.

\section{References}

1. Zhang Qiang, A numerical study of Richtmyer-Meshkov instability driven by cylindrical shock, Physics of Fluids, 1998, 10(4): 947-993.

2. Cloutman, L. D., Wehner, M. E., Numerical simulation of Richtmyer-Meshkov instabilities, Phys. Fluids A, 1992, 4: 1821

3. Youngs, D. L., Numerical simulation of mixing by Rayleigh-Taylor and Richtmyer-Meshkov instabilities, Laser Part. Beams, 1994, 12: 725.

4. Cohen, R. H., Dannevik, W. P., Dimits, A. M. et al, Three-dimensionl simulation of a RichtmyerMeshkov instability with a two-scale initial perturbation, Phys. Fluids, 2002, 14(10): 3692.

5. Ma Yanwen, Fu Dexun, Fouth order accurate compact scheme with group velocity control (GVC), Science in China, Ser. A, 2001, 44(9): 1197-1204. 